\title{
L'inflammation postprandiale : les données récentes suggèrent un rôle préventif des protéines alimentaires et de leur nature
}

\author{
François MARIOTTI \\ Unité 914, \\ AgroParisTech, \\ INRA, \\ Physiologie de la nutrition \\ et du comportement alimentaire, \\ 16 rue Claude Bernard, \\ 75005 Paris; \\ UFR de Biologie et nutrition humaines, \\ AgroParisTech, \\ 16 rue Claude Bernard, \\ 75005 Paris, \\ France \\ <francois.mariotti@agroparistech.fr >
}

\begin{abstract}
It is now well known that a high-energy meal that is rich in saturated fat and sugars induces the transient appearance of some metabolic dysregulations and inflammatory-related phenomena in the postprandial state, in close association with the increase in plasma triglycerides and glucose. Documented features of postprandial inflammation include the increase in plasma inflammatory markers related to the systemic level (e.g. proinflammatory cytokines) or the vascular endothelium (e.g. adhesion molecules), the activation of blood leucocytes, and different physiological markers of "endothelial dysfunction", which relates to the transition of the endothelium toward a pro-inflammatory atherogenic phenotype. The role of fat and carbohydrates in postprandial inflammation has been much studied, while the impact of dietary protein has been overlooked. The present review will focus on the few recent studies that have examined the effect of dietary protein, and different dietary protein sources, on postprandial inflammation and reported a favorable impact on endothelial function. The literature also showed that arginine and cysteine are important amino acids in mediating these effects. Some further studies in this setting are warranted to better characterize and analyze the potential benefit of different dietary protein sources for cardiovascular risk.
\end{abstract}

Key words: postprandial period, dietary proteins, protein quality, low-grade inflammation, vascular endothelial function, nutrition, metabolic syndrome, arginine

traditionnellement établis. La CRP est une protéine classique de la phase aiguë, qui signe traditionnellement la réponse inflammatoire classique. Des élévations minimes de la CRP, par rapport à la réponse inflammatoire de forte amplitude, correspondent à la notion $d^{\prime}$ " inflammation à bas bruit », c'est-à-dire une situation inflammatoire qui n'est pas associée aux manifestations cliniques de I'inflammation traditionnelle.

L'inflammation à bas bruit se présente souvent conjointement à d'autres facteurs de risque de maladies cardiovasculaires que l'on regroupe sous la dénomination de "Syndrome métabolique " (Ford et al., 2005). D'un point de vue clinique, le syndrome métabolique correspond à la présentation conjointe de certaines anomalies que sont l'obésité abdominale, une élévation de triglycéridémie et de la glycémie à jeun, une faible concentration plasmatique en cholestérol-HDL et une élévation de la pression artérielle (Grundy et al., 2004). La prévalence du syndrome métabolique est en forte augmentation dans les pays industrialisés, touchant actuellement un tiers de la population états-unienne et $10 \%$ de la population française (Ford et al., 2004; Ford et al., 2010; Rousseau et Sévin, 2009). Bien que cliniquement hétérogène, le syndrome métabolique peut se définir d'un point de vue physiopathologique comme la manifestation d'une résistance à l'insuline multitissus se développant dans un contexte d'inflammation à bas bruit, étroitement associé à l'apparition de dysfonctions

Pour citer cet article : Mariotti F. L'inflammation postprandiale : les données récentes suggèrent un rôle préventif des protéines alimentaires et de leur nature. OCL $2011 ; 18(1)$ : 14-20. doi : 10.1684/ocl.2011.0366 
de I'endothélium vasculaire. L'obésité viscérale est un contributeur physiopathologique très important, notamment bien associé à l'inflammation à bas bruit.

Ainsi, pratiquement, I'inflammation à bas bruit accompagne étroitement la résistance à l'insuline et à la dysfonction endothéliale vasculaire. Physiopathologiquement, la dysfonction endothéliale correspond à l'expression d'un phénotype vasculaire proathérogène, avec une composante inflammatoire principale. Aussi peut-on considérer que la dysfonction endothéliale correspond à la manifestation de la composante vasculaire d'un phénomène général inflammatoire à bas bruit (Sattar, 2004). La dysfonction endothéliale est ainsi le phénomène objectivable de l'initiation de la maladie inflammatoire qu'est l'athérosclérose.

Pour caractériser I'inflammation à basbruit, la CRP est le marqueur de référence, mais on peut également avoir recours à d'autres protéines de la phase aiguë, ou s'intéresser aux médiateurs inflammatoires, les cytokines et chimiokines (pro)inflammatoires. L'IL-6, une cytokine clé pro-inflammatoire, est notamment bien associée à la CRP et à I'adiposité (Browning et al., 2008). Pour les raisons évoquées plus haut, les marqueurs de fonction endothéliale sont également pertinents, notamment quand il s'agit de médiateurs biologiquement impliqués dans la réaction inflammatoire locale des vaisseaux (glycoprotéines d'adhésion cellulaires : ICAM-1, VCAM-1, Sélectine-E). Les mesures de fonction endothéliale qui sont étroitement associées à une diminution de la production ou de la biodisponibilité de monoxyde d'azote (la molécule clé de la régulation de la physiologie de l'endothélium) sont également pertinentes. C'est le cas de la capacité vasodilatatoire artérielle dans le cadre du contrôle local, testée en réponse à un stimulus physiologique (hyperhémie réactive) ou l'administration d'un agoniste (acétylcholine). À ce titre, I'inflammation à bas-bruit systémique est associée à ces mesures de la fonction endothéliale chez l'homme (Cleland et al., 2000; Kusche-Vihrog et al., in press), et différentes données, essentiellement chez l'animal ou selon des modèles cellulaires, indiquent que les cytokines proinflammatoires et la CRP produisent des anomalies de production/biodisponibilité du monoxyde $d^{\prime}$ 'azote, et d'autres voies de dysfonction endothéliale (Clapp et al., 2005; Fichtlscherer et al., 2004; Guzik et al., 2006; Schwartz et al., 2007).

\section{Manifestations inflammatoires en phase postprandiale}

La phase postprandiale est une phase critique de gestion métabolique de I'afflux de nutriments. En effet, lors de cette phase, la dynamique métabolique doit permettre de satisfaire aux principes de l'homéostasie tout en permettant une utilisation optimale de l'arrivée des nutriments. Cette complexité dynamique, qui relève de processus homéodynamiques, intéresse les nutritionnistes depuis longtemps.

Plus récemment, au cours de la dernière décennie, il a été établi que de fortes provocations métaboliques avec des repas riches en acides gras saturés et en sucres simples entrainent l'apparition transitoire de manifestations d'inflammation à bas bruit et de dysfonction endothéliale (par exemple: (Alipour et al., 2007; Bae et al., 2003; Blackburn et al., 2006; Burdge and Calder, 2005; Ceriello, 2000; de Koning and Rabelink, 2002; Lee et al., 2002; Nappo et al., 2002; Poppitt, 2005; van Oostrom et al., 2003; Vogel et al., 1997; Wautier et al., 2006)). L'ampleur et la chronologie d'apparition de ces phénomènes sont étroitement liées à l'excursion lipémique et glycémique postprandiale (Wautier et al., 2006; Alipour et al., 2008a; Bavenholm and Efendic, 2006; Ceriello, 2004; van Oostrom et al., 2004). L'inflammation postprandiale, décrite au niveau systémique (Blackburn et al., 2006; Ceriello et al., 2004), a été rapportée au niveau des leucocytes circulants (van Oostrom et al., 2004; Aljada et al., 2004), du tissu adipeux viscéral (Magne et al., 2010), et bien sûr au niveau vasculaire, soit comme l'augmentation des concentrations circulantes en molécules d'adhésion à I'endothélium (ICAM-1 et VCAM-1, (Nappo et al., 2002)), soit comme l'altération de fonctions plus larges ou intégratives relevant de la "fonction endothéliale » et attestant de l'activation de nature inflammatoire de l'endothélium. II convient néanmoins de signaler que certaines études postprandiales sont négatives, c'est-à-dire qu'elles n'ont pas rapporté d'augmentation de marqueurs usuels d'inflammation systémique (par exemple : IL-6 et les marqueurs d'adhésion (Campbell et al., 2006; Poppitt et al., 2008; Tsai et al., 2004)).

Les mécanismes qui président à l'apparition de l'inflammation à bas bruit sont encore mal identifiés. L'augmentation massive des concentrations en glucose et en triglycérides (tout particulièrement les chylomicrons et leur remnants) semble bien capable d'induire une activation des voies de signalisation inflammatoire au niveau leucocytaire, endothélial, et même du tissu adipeux viscéral (Wautier et al., 2006; Magne et al., 2010; Alipour et al., 2008b; Dickinson et al., 2008; Norata et al., 2007). Par ailleurs, I'apparition d'un stress oxydant pourrait être un important contributeur à l'inflammation postprandiale (Bowen and Borthakur, 2004; Sies et al., 2005). En effet, sous l'angle moléculaire, des voies majeures d'activation de la réponse inflammatoire sont réputés très sensibles au statut redox, notamment la voie NF- $\kappa B$. Au niveau vasculaire, la production/biodisponibilité du monoxyde d'azote est bien connue pour être très sensible au statut redox (Bowen and Borthakur, 2004). Surtout, un assez grand nombre d'études rapporte une augmentation du stress oxydant après une charge alimentaire (Tsai et al., 2004; Devaraj et al., 2008), en étroite relation temporelle avec l'apparition des phénomènes inflammatoires (Bae et al., 2001; Tushuizen et al., 2006). Certaines études n'ont pas rapporté de stress oxydant postprandial, mais cela pourrait s'expliquer par un choix de marqueurs insuffisamment sensibles, ou du fait que les signes du stress oxydant au niveau systémique (c'est-à-dire évalué à partir du plasma) sont intrinsèquement faibles. Un élément important en faveur de l'implication du stress oxydant dans I'inflammation postprandiale est fourni par les différentes études qui ont montré qu'un prétraitement ou la co-administration de fortes doses de vitamines et/ou de substances antioxydantes réduisait la dysfonction endothéliale et l'expression de certains marqueurs inflammatoires dans la phase postprandiale (Nappo et al., 2002; Bae et al., 2001; Anderson et al., 2006; Neri et al., 2005).

Enfin, signalons que l'ensemble de ces phénomènes inflammatoires postprandiaux est d'autant plus patent que les sujets présentent un syndrome métabolique et des dérèglements apparents de I’homéostasie (Patel et al., 2007). 
Au final, on voit que les phénomènes inflammatoires postprandiaux présentent une frappante congruence en termes de physiopathologie nutritionnelle : ces réactions indésirables sont connues pour être impliquées dans l'initiation et la progression du syndrome métabolique ; elles apparaissent en relation avec le degré de perturbation homéostatique induit par l'ingestion des nutriments énergétiques, et en association avec des phénomènes de dérégulation à l'échelle biochimique et cellulaire ; et, enfin, ils sont d'autant plus marqués que le sujet présente au préalable un moindre capacité de régulation métabolique, associée à des facteurs de risque de maladies cardiovasculaires et diabétiques. En retour, pour un individu, l'ampleur de la triglycéridémie et de la glycémie postprandiale après un repas de charge est considérée comme un facteur de risque cardiovasculaire et diabétique, particulièrement prédictif (Cohn, 2008; Lairon et al., 2007; O'Keefe and Bell, 2007). Ainsi, le paradigme actuel est que la répétition de ces événements transitoires et silencieux lors des phases postprandiales pourrait être un mécanisme d'initiation et de progression des dérégulations métaboliques et des maladies cardiovasculaires et diabétiques (Burdge and Calder, 2005; O'Keefe and Bell, 2007).

L'étude des dérégulations et dysfonctionnements postprandiaux fournit donc un modèle très intéressant pour l'étude de l'influence des nutriments sur le risque métabolique et ses répercussions inflammatoires. Le rôle des acides gras et des glucides alimentaires, et en particulier l'importance de leur nature, a été largement étudié. Le lecteur pourra se reporter à quelques articles de synthèse (par exemple: (Poppitt, 2005; Dandona et al., 2010)) et articles originaux importants (par exemple : (Bellido et al., 2004; Cortes et al., 2006; Jimenez-Gomez et al., 2009; Vogel et al., 2000; West et al., 2005a)).

\section{Des hypothèses pour un effet favorable des protéines alimentaires}

À l'inverse, l'influence des protéines sur l'apparition de l'inflammation postprandiale a été peu étudiée. Connaitre I'influence des protéines sur l'initiation des phénomènes indésirables postpran- diaux pourrait pourtant permettre de raisonner l'importance de la quantité et surtout de la qualité des protéines alimentaires dans I'initiation physiopathologique nutritionnelle. En termes de qualité nutritionnelle, il a déjà été souligné que les critères classiques, relatifs à la participation des protéines au métabolisme protéique, n'étaient pas assez pertinents, et insuffisamment discriminants, en termes de santé pour la population adulte en bonne santé d'un pays industrialisé comme la France ; il faut donc étudier le rôle des protéines dans I'initiation et la progression des maladies qui affectent ces populations (Debry, 2004; Mariotti, 2003; Mariotti and Tome, 2003).

En outre, la littérature fournit des hypothèses concernant l'influence que certains acides aminés pourraient avoir sur l'initiation de phénomènes inflammatoires. En particulier, deux acides aminés, l'arginine et la cystéine, sont de possibles candidats pour une modulation de l'inflammation systémique et vasculaire. Ces deux acides aminés ne sont pas strictement indispensables, au sens classique du terme, c'est-à-dire qu'ils ne sont pas requis dans I'alimentation pour assurer un renouvellement normal des protéines corporelles (Mariotti and Tome, 2003; AFSSA, 2008). En revanche, les autres voies métaboliques de leur utilisation impliquent des composés dont l'importance intrinsèque, biologique, est très grande. Sommairement, la cystéine est le précurseur critique de la synthèse du glutathion, qui est le principal tampon redox intracellulaire et participe de façon essentielle, directement et indirectement, aux défenses antioxydantes (Lu, 2009). Il est probablement impliqué dans la production/biodisponibilité du monoxyde d'azote. Sur cette base, de nombreux travaux ont cherché à déterminer l'influence de l'apport en cystéine sur le stress oxydant et les dérégulations métaboliques. Dans le cadre d'un exercice physique (qui est un bon modèle de provocation prooxydante physiologique) et dans différentes conditions, et il a été rapporté, chez l'animal et chez l'homme, que la consommation de cystéine, à dose nutritionnelle, modifie le statut en glutathion (Huneau et al., 2008; Mariotti et al., 2004; Parthimos et al., 2007; Tsakiris et al., 2006; Wu et al., 2004). Puis, nous avons montré que l'apport en cystéine chez le rat réduisait l'apparition du stress oxydant et de l'insulino-résistance induite par un régime riche en sucre chez I'animal (Blouet et al., 2007a). L'effet de la cystéine semble s'exercer de façon aiguë, c'est-à-dire dès la phase postprandiale (Blouet et al., 2006) et s'accompagne d'une modulation de la biodisponibilité du monoxyde d'azote (Blouet et al., 2007b). Pour une revue récente assez complète en langue française, le lecteur pourra se rapporter à Huneau et al., 2008. D'autre part, l'arginine, en tant que précurseur du monoxyde d'azote a attiré depuis longtemps l'intérêt des chercheurs. L'administration d'arginine est bien connue pour modifier les nombreuses fonctions associées au monoxyde d'azote (Loscalzo, 2000; Ohta et al., 2007; Preli et al., 2002; West et al., 2005b; Wu and Meininger, 2000). Chez l'homme et I'animal, de nombreuses études ont rapporté un effet bénéfique d'un apport oral en arginine sur la fonction endothéliale et l'expression des molécules d'adhésion (Preli et al., 2002). Certaines études ont mis en évidence les effets bénéfiques de l'arginine à court/moyen terme, à dose alimentaire, et même dans un contexte de stress nutritionnel (Marchesi et al., 2001). Cependant, I'essentiel du corpus scientifique a été obtenu en situation de risque ou de maladie, et à des niveaux d'apport en arginine supérieur aux doses nutritionnelles. Au final, comme la situation postprandiale crée temporairement une situation mimant celle de sujets à risque ou malade, impliquant du stress oxydant, on peut faire I'hypothèse que l'apport en arginine et en cystéine pendant le repas pourrait avoir des répercussions sur l'ampleur des phénomènes délétères postprandiaux, et notamment les phénomènes inflammatoires, systémiques et vasculaires.

\section{Une influence de la quantité de protéines}

L'importance des protéines au cours de cette phase postprandiale a été clairement montrée pour la première fois par l'équipe de Sabine Westphal (2006). Dans cette étude, les sujets ont consommé, selon un dispositif croisé, une forte charge lipidique, seule ou additionnée de protéines $(50 \mathrm{~g}$ de caséinates de sodium, ou de protéines de soja) et la fonction endothéliale (estimée par l'ampleur de la dilatation brachiale post-ischémique) a 
été mesurée toutes les heures pendant huit heures après ingestion. La charge lipidique seule a bien induit une forte dépression de la fonction endothéliale, tandis que l'ajout de protéines au repas permettait de maintenir inaltérée la fonction endothéliale tout au long de la période postprandiale. Autrement dit, I'adjonction de protéines au repas de charge lipidique a prévenu l'apparition de la dysfonction endothéliale postprandiale. Les auteurs ont formulé différentes hypothèses pour expliquer ce résultat. Tout d'abord, les auteurs ont constaté que l'adjonction de protéines avait très fortement majoré l'excursion insulinique postprandiale. Comme l'insuline a un effet direct favorable sur la fonction endothéliale, I'effet favorable des protéines pourrait s'expliquer en grande partie par leur effet insulinotrope. En outre, un effet sur la sécrétion et l'action de I'insuline pourrait également expliquer la modération de l'apparition de l'excursion lipémique proathérogène. Les auteurs ont aussi proposé que l'apport d'arginine par les protéines puisse expliquer la préservation de la fonction endothéliale. Cette hypothèse a été étayée par la suite par cette même équipe, qui a montré que le seul ajout de 2,5 grammes d'arginine au repas lipidique suffisait à prévenir l'altération postprandiale de la fonction endothéliale (Borucki et al., 2009), confirmant un résultat précédent obtenu avec une dose $\mathrm{d}^{\prime}$ arginine beaucoup plus forte (Lin et al., 2008).

Nous avons montré incidemment qu'un mélange (50 g) d'acides aminés n'induisait pas de dysfonction endothéliale, ni l'apparition des molécules d'adhésion dans la phase postpandiale (Mariotti et al., 2007), mais l'étude de Westphall et al. a permis de montrer pour la première fois que les protéines alimentaires, données à forte dose, non seulement n'exercent pas les effets délétères des acides gras (saturés) et des sucres mais peuvent au contraire prévenir ces effets.

Néanmoins, cette étude permet surtout une interprétation en termes de quantité protéique. En effet, la quantité importante de protéines ajoutées $(50 \mathrm{~g})$ était élevée par rapport à la charge lipidique ( $70 \mathrm{~g})$, si bien que l'introduction des protéines a influencé, de façon probablement importante, la vidange gastrique du repas, ainsi que l'insulinémie. En somme, le repas, ainsi "rééquilibré » énergétiquement a pu entrainer une moindre exposition de l'organisme à l'arrivée massive des acides gras du repas, comme cela a d'ailleurs été rapporté par ailleurs par les mêmes auteurs (Westphal et al., 2004). L'excursion triglycéridémique était ainsi moins importante et plus étalée dans le temps (Westphal et al., 2006). Les effets des protéines pourraient donc aussi s'expliquer par une moindre provocation métabolique par les acides gras du régime, tout autant que par une atténuation des effets des acides gras sur le stress oxydant et l'inflammation.

\section{Une influence de la nature des protéines}

En ce qui concerne l'importance de la qualité protéique, c'est-à-dire de la nature des protéines alimentaires, les éléments sont plus récents encore. Notre équipe a développé un modèle d'inflammation postprandiale chez le rat induit par un repas de charge composé d'acides gras saturés (huile de palme, $60 \%$ de l'énergie du repas), de sucre (saccharose, $20 \%$ de l'énergie) et incluant des protéines (caséines, $20 \%$ de l'énergie). Un travail préalable nous a permis d'explorer les caractéristiques de ce modèle : la phase postprandiale s'accompagne de l'apparition d'un stress oxydant systémique et vasculaire, d'une inflammation à bas bruit, identifiée au niveau systémique (cytokines proinflammatoires circulantes), au niveau des leucocytes circulants (activation des neutrophiles) et du tissu adipeux viscéral, et d'une dysfonction endothéliale (Magne et al., 2010; Magne et al., 2009). En utilisant ce modèle, nous avons pu montrer que la substitution des caséines par des protéines de colza prévenait l'apparition de la dysfonction endothéliale postprandiale, sans modifier l'excursion lipémique (Magne et al., 2009). Les protéines de colza ont également spécifiquement atténué le stress oxydant systémique, et considérablement réduit le stress oxydant vasculaire. Nous avons en outre constaté que la seule adjonction d'arginine et de cystéine, à hauteur de ce qui était apporté par les protéines de colza, mimait en grande partie l'effet des protéines de colza. En revanche, ces effets favorables ne s'accompagnent pas de modulation de la forte augmentation postprandiale des cytokines proinflammatoires circulantes.

\section{De la difficulté d'étudier I'inflammation postprandiale et d'analyser l'influence des protéines}

En revanche, très récemment, une équipe n'a pas identifié d'effet favorable de la manipulation des protéines dans des repas provoquant une inflammation postprandiale. Dans cette étude, la substitution de $50 \mathrm{~g}$ de sucre au sein du repas de charge par $50 \mathrm{~g}$ de protéines, sous forme de caséine ou de protéines solubles du lait n'a pas modifié significativement I'augmentation postprandiale des concentrations plasmatiques en IL-6 (Pal and Ellis, 2011). En outre, une difficulté d'interprétation des différents résultats d'inflammation postprandiale est liée à la variété des repas de charge (niveau énergétique, composition nutritionnelle, présentation) et des critères d'évaluation de l'inflammation (type de marqueurs, compartiments et tissus étudiés, etc.). Cette difficulté est constante lors de l'emploi de tests de provocation alimentaire chez I'homme (Lairon et al., 2007) et les difficultés s'accroissent dans le cadre de la réponse inflammatoire. Aussi, une réflexion importante est à mener pour bien choisir les critères d'évaluation de l'inflammation postprandiale qui serviront de critère principal pour l'évaluation de l'effet des protéines. On peut par exemple noter que les études utilisant les cytokines inflammatoires comme marqueurs ont eu des résultats très variables, et souvent discordants : la concentration de certaines cytokines (comme TNF- $\alpha$ ) ou protéines de I'inflammation (CRP) ne semble pas augmenter après la plupart des repas de charge utilisés, et l'augmentation postprandiale d'IL-6 chez I'homme sain n'est pas retrouvée par tous les auteurs (Mariotti et al., données personnelles, (Campbell et al., 2006; Poppitt et al., 2008; Tsai et al., 2004)). De façon générale, il est probable que les marqueurs systémiques soient moins robustes pour ce type d'étude et qu'il faille plutôt essayer de caractériser l'activation des voies inflammatoires au niveau tissulaire, comme cela a pu être fait chez I'homme au niveau des leucocytes circulants (Patel et al., 2007; Ghanim et al., 2010).

L'inflammation postprandiale se présente vraisemblablement de façon 
hétérogène et les mécanismes et les tissus impliqués dans son initiation sont encore mal identifiés, mais probablement très nombreux et interactifs (Burdge and Calder, 2005; Poppitt, 2005; Ghanim et al., 2010; Ceriello and Motz, 2004; Patel et al., 2009; Wang et al., 2009). On ne sait presque rien sur les mécanismes qui soustendent l'effet favorable de la quantité et de la qualité des protéines. Cependant, comme nous l'avons vu plus haut, les quelques résultats dont nous disposons permettent de renforcer les hypothèses sur les effets de certains acides aminés (arginine et cystéine), ce qui permet de suggérer ainsi des pistes de recherche attrayantes.

Pour finir, il convient de dire un mot sur le fait que les protéines, ou plutôt les "matières protéiques", peuvent également avoir une influence sur l'inflammation postprandiale en agissant très en amont, c'est-à-dire sur la mise à disposition des acides gras et l'excursion triglycéridémique qui en résulte. Comme nous l'avons vu, c'est une hypothèse pour interpréter l'effet quantitatif de protéines dans un repas de charge, tel que mis en évidence par Westphal et al. (Westphal et al., 2004; Westphal et al., 2006), mais l'importance de ces phénomènes a été soulignée par les résultats d'études récentes de la littérature (Mortensen et al., 2009; Pal et al., in press)) et de notre équipe (Mariotti et al., données non publiées). Une partie des effets des matières protéiques pourrait provenir de leurs propriétés physicochimiques et de leurs effets sur les caractéristiques structurelles du repas, les interactions entre protéines et lipides dans le repas et lors des phases de digestion. Pratiquement, il convient de ne pas négliger ces phénomènes si l'on s'intéresse à la réalité des conséquences de la manipulation de la quantité et de la nature des protéines dans les aliments et les régimes, en situation " réelle ".

\section{RÉFÉRENCES}

AFSSA. Apport en protéines : consommation, qualité, besoins et recommandations. AFSSA, 2008.

Alipour A, Elte JW, van Zaanen HC, Rietveld AP, Cabezas MC. Postprandial inflammation and endothelial dysfuction. Biochem Soc Trans 2007 ; 35 : 466-9.
Alipour A, Elte JW, van Zaanen HC, Rietveld AP, Castro Cabezas M. Novel aspects of postprandial lipemia in relation to atherosclerosis. Atheroscler Suppl 2008 ; 9 : 39-44.

Alipour A, van Oostrom AJ, Izraeljan A, et al. Leukocyte activation by triglyceride-rich lipoproteins. Arterioscler Thromb Vasc Biol 2008 ; 28 : 792-7.

Aljada A, Mohanty P, Ghanim H, et al. Increase in intranuclear nuclear factor kappaB and decrease in inhibitor kappaB in mononuclear cells after a mixed meal: evidence for a proinflammatory effect. Am / Clin Nutr $2004 ; 79$ : 682-90.

Anderson RA, Evans LM, Ellis GR, et al. Prolonged deterioration of endothelial dysfunction in response to postprandial lipaemia is attenuated by vitamin C in Type 2 diabetes. Diabet Med 2006 ; 23 : 258-64.

Bae JH, Bassenge E, Kim KB, et al. Postprandial hypertriglyceridemia impairs endothelial function by enhanced oxidant stress. Atherosclerosis $2001 ; 155$ : 517-23.

Bae JH, Schwemmer M, Lee IK, et al. Postprandial hypertriglyceridemia-induced endothelial dysfunction in healthy subjects is independent of lipid oxidation. Int J Cardiol $2003 ; 87$ : 259-67.

Bavenholm PN, Efendic S. Postprandial hyperglycaemia and vascular damage-the benefits of acarbose. Diab Vasc Dis Res 2006 ; $3: 72-9$.

Bellido C, Lopez-Miranda J, Blanco-Colio LM, et al. Butter and walnuts, but not olive oil, elicit postprandial activation of nuclear transcription factor kappaB in peripheral blood mononuclear cells from healthy men. Am J Clin Nutr $2004 ; 80$ : 1487-91.

Blackburn P, Despres JP, Lamarche B, et al. Postprandial variations of plasma inflammatory markers in abdominally obese men. Obesity (Silver Spring) 2006 ; 14 : 1747-54.

Blouet C, Mariotti F, Mikogami T, Tome D, Huneau JF. Meal cysteine improves postprandial glucose control in rats fed a highsucrose meal. J Nutr Biochem 2006 ; 18 : 519 24.

Blouet C, Mariotti F, Azzout-Marniche D, et al. Dietary cysteine alleviates sucrose-induced oxidative stress and insulin resistance. Free Radic Biol Med 2007 ; 42 : 1089-97.

Blouet C, Mariotti F, Mathe V, Tome D, Huneau JF. NO bioavailability and not production is first altered during the onset of insulin resistance in sucrose-fed rats Exp Biol Med (Maywood) 2007 ; 232 : 1458-64.

Borucki K, Aronica S, Starke I, Luley C, Westphal S. Addition of $2.5 \mathrm{~g} \mathrm{~L}$-arginine in a fatty meal prevents the lipemia-induced endothelial dysfunction in healthy volunteers. Atherosclerosis 2009 ; 205 : 251-4.
Bowen PE, Borthakur G. Postprandial lipid oxidation and cardiovascular disease risk. Curr Atheroscler Rep 2004 ; 6 : 477-84.

Browning LM, Krebs JD, Magee EC, Fruhbeck $\mathrm{G}$, Jebb SA. Circulating markers of inflammation and their link to indices of adiposity. Obes Facts 2008 ; 1 : 259-65.

Burdge GC, Calder PC. Plasma cytokine response during the postprandial period: a potential causal process in vascular disease? Br J Nutr 2005 ; 93 : 3-9.

Campbell CG, Brown BD, Dufner D, Thorland WG. Effects of soy or milk protein during a high-fat feeding challenge on oxidative stress, inflammation, and lipids in healthy men. Lipids 2006 ; 41 : 257-65.

Ceriello A. The post-prandial state and cardiovascular disease : relevance to diabetes mellitus. Diabetes Metab Res Rev 2000 ; 16 : 125-32.

Ceriello A. Impaired glucose tolerance and cardiovascular disease: the possible role of post-prandial hyperglycemia. Am Heart J $2004 ; 147$ : 803-7.

Ceriello A, Motz E. Is oxidative stress the pathogenic mechanism underlying insulin resistance, diabetes, and cardiovascular disease? The common soil hypothesis revisited. Arterioscler Thromb Vasc Biol $2004 ; 24$ : 816-23.

Ceriello A, Quagliaro L, Piconi L, et al. Effect of postprandial hypertriglyceridemia and hyperglycemia on circulating adhesion molecules and oxidative stress generation and the possible role of simvastatin treatment. Diabetes 2004 ; 53 : 701-10.

Clapp BR, Hirschfield GM, Storry C, et al. Inflammation and endothelial function: direct vascular effects of human C-reactive protein on nitric oxide bioavailability. Circulation $2005 ; 111$ : 1530-6.

Cleland SJ, Sattar N, Petrie JR, Forouhi NG, Elliott HL, Connell JM. Endothelial dysfunction as a possible link between C-reactive protein levels and cardiovascular disease. Clin Sci (Lond) 2000 ; 98 : 531-5.

Cohn JS. Are we ready for a prospective study to investigate the role of chylomicrons in cardiovascular disease? Atheroscler Suppl $2008 ; 9: 15-8$.

Cortes B, Nunez I, Cofan M, et al. Acute effects of high-fat meals enriched with walnuts or olive oil on postprandial endothelial function. J Am Coll Cardiol 2006 ; 48 : 1666-71.

Dandona P, Ghanim H, Chaudhuri A, Dhindsa S, Kim SS. Macronutrient intake induces oxidative and inflammatory stress: potential relevance to atherosclerosis and insulin resistance. Exp Mol Med 2010; 42 : 245-53. 
de Koning EJ, Rabelink TJ. Endothelial function in the post-prandial state. Atheroscler Suppl $2002 ; 3: 11-6$.

Debry G. Chapter 4 - Data on Atherosclerosis. In: Dietary Proteins and Atherosclerosis. CRC Press ; 2004.

Devaraj S, Wang-Polagruto J, Polagruto J, Keen CL, Jialal I. High-fat, energy-dense, fastfood-style breakfast results in an increase in oxidative stress in metabolic syndrome. Metabolism 2008 ; 57 : 867-70.

Dickinson S, Hancock DP, Petocz P, Ceriello A, Brand-Miller J. High-glycemic index carbohydrate increases nuclear factor-kappaB activation in mononuclear cells of young, lean healthy subjects. Am J Clin Nutr 2008 ; 87 : 1188-93.

Fichtlscherer S, Breuer S, Schachinger V, Dimmeler S, Zeiher AM. C-reactive protein levels determine systemic nitric oxide bioavailability in patients with coronary artery disease. Eur Heart / 2004 ; 25 : 1412-8.

Ford ES, Giles WH, Mokdad AH. Increasing prevalence of the metabolic syndrome among u.s Adults. Diabetes Care 2004 ; 27 : 2444-9.

Ford ES, Ajani UA, Mokdad AH. The metabolic syndrome and concentrations of $\mathrm{C}$ reactive protein among U.S. youth. Diabetes Care $2005 ; 28: 878-81$.

Ford ES, Li C, Zhao G. Prevalence and correlates of metabolic syndrome based on a harmonious definition among adults in the US. J Diabetes $2010 ; 2$ : 180-93.

Ghanim H, Sia CL, Upadhyay M, et al. Orange juice neutralizes the proinflammatory effect of a high-fat, high-carbohydrate meal and prevents endotoxin increase and Toll-like receptor expression. Am / Clin Nutr 2010 ; 91 : 940-9.

Grundy SM, Brewer Jr HB, Cleeman JI, Smith Jr SC, Lenfant C. Definition of metabolic syndrome: report of the National Heart, Lung, and Blood Institute/American Heart Association conference on scientific issues related to definition. Arterioscler Thromb Vasc Biol 2004 ; 24 : e13-8.

Guzik TJ, Mangalat D, Korbut R. Adipocytokines - novel link between inflammation and vascular function? J Physiol Pharmacol 2006 ; $57: 505-28$.

Huneau JF, Mariotti F, Blouet C, Tome D, Blachier F. Implications métaboliques, physiologiques et fonctionnelles de I'apport en acides aminés soufrés et en protéines riches en acides aminés soufrés. In : Roberfroid $M$, Coxam V, Delzenne N, editors. Aliments fonctionnels (2ieme édition). Paris : Lavoisier ; 2008.

Jimenez-Gomez Y, Lopez-Miranda J, BlancoColio LM, et al. Olive oil and walnut breakfasts reduce the postprandial inflammatory response in mononuclear cells compared with a butter breakfast in healthy men. Atherosclerosis 2009 ; 204 : e70-6.

Kusche-Vihrog K, Urbanova K, Blanque A, et al. $\mathrm{C}$-reactive protein makes human endothelium stiff and tight. Hypertension 57: 231-7.

Lairon D, Lopez-Miranda J, Williams C. Methodology for studying postprandial lipid metabolism. Eur J Clin Nutr 2007 ; 61 : 1145 61.

Lee IK, Kim HS, Bae JH. Endothelial dysfunction: its relationship with acute hyperglycaemia and hyperlipidemia. Int / Clin Pract Suppl 2002 : 59-64.

Lin CC, Tsai WC, Chen JY, Li YH, Lin LJ, Chen $\mathrm{JH}$. Supplements of L-arginine attenuate the effects of high-fat meal on endothelial function and oxidative stress. Int / Cardiol 2008 ; 127 : 337-41.

Loscalzo J. What we know and don't know about L-arginine and NO. Circulation 2000 ; 101 : 2126-9.

Lu SC. Regulation of glutathione synthesis. Mol Aspects Med 2009 ; 30 : 42-59.

Magne J, Huneau JF, Delemasure S, Rochette L, Tome D, Mariotti F. Whole-body basal nitric oxide production is impaired in postprandial endothelial dysfunction in healthy rats. Nitric Oxide 2009 ; 21 : 37-43.

Magne J, Mariotti F, Fischer R, Mathe V, Tome D, Huneau JF. Early postprandial low-grade inflammation after high-fat meal in healthy rats : possible involvement of visceral adipose tissue. J Nutr Biochem 2010 ; 21 : 550-5.

Marchesi S, Lupattelli G, Siepi D, et al. Oral Larginine administration attenuates postprandial endothelial dysfunction in young healthy males. J Clin Pharm Ther 2001 ; 26 : 343-9.

Mariotti F. Analyse d'ouvrage: G. Debry. Dietary Protein and Atheroslerosis \{CRC Press, novembre 2003\}. Sci Alim $2003 ; 23$ : 717-8.

Mariotti F, Tome D. New data to appreciate the quality of vegetal proteins in humans Implications and outlook. Ocl-Oleagineux Corps Gras Lipides 2003 ; 10 : 17-22.

Mariotti F, Simbelie KL, Makarios-Lahham L, et al. Acute ingestion of dietary proteins improves post-exercise liver glutathione in rats in a dose-dependent relationship with their cysteine content. J Nutr $2004 ; 134$ : 128-31.

Mariotti F, Huneau JF, Szezepanski I, et al. Meal amino acids with varied levels of arginine do not affect postprandial vascular endothelial function in healthy young men. J Nutr 2007 ; 137 : 1383-9.

Mortensen LS, Hartvigsen ML, Brader LJ, et al. Differential effects of protein quality on postprandial lipemia in response to a fat-rich meal in type 2 diabetes: comparison of whey, casein, gluten, and cod protein. $A m$ J Clin Nutr 2009; $90:$ 41-8.

Nappo F, Esposito K, Cioffi M, et al. Postprandial endothelial activation in healthy subjects and in type 2 diabetic patients: role of fat and carbohydrate meals. J Am Coll Cardiol $2002 ; 39: 1145-50$.

Neri S, Signorelli SS, Torrisi B, et al. Effects of antioxidant supplementation on postprandial oxidative stress and endothelial dysfunction : a single-blind, 15-day clinical trial in patients with untreated type 2 diabetes, subjects with impaired glucose tolerance, and healthy controls. Clin Ther $2005 ; 27$ : 1764-73.

Norata GD, Grigore L, Raselli S, et al. Postprandial endothelial dysfunction in hypertriglyceridemic subjects: molecular mechanisms and gene expression studies. Atherosclerosis 2007 ; 193 : 321-7.

Ohta F, Takagi T, Sato H, Ignarro LJ. Low-dose $\mathrm{L}$-arginine administration increases microperfusion of hindlimb muscle without affecting blood pressure in rats. Proc Natl Acad Sci U S A 2007 ; 104 : 1407-11.

O'Keefe JH, Bell DS. Postprandial hyperglycemia/hyperlipidemia (postprandial dysmetabolism) is a cardiovascular risk factor. Am J Cardiol $2007 ; 100$ : 899-904.

Pal S, Ellis V. Acute effects of whey protein isolate on blood pressure, vascular function and inflammatory markers in overweight postmenopausal women. Br J Nutr 2011 Jan 28 : 1-8 [Epub ahead of print].

Pal S, Ellis V, Ho S. Acute effects of whey protein isolate on cardiovascular risk factors in overweight, post-menopausal women. Atherosclerosis 212 : 339-44.

Parthimos T, Tsopanakis C, Angelogianni P, Schulpis KH, Parthimos N, Tsakiris S. LCysteine supplementation prevents exercise-induced alterations in human erythrocyte membrane acetylcholinesterase and $\mathrm{Na}$ $(+), \mathrm{K}(+)$-ATPase activities. Clin Chem Lab Med 2007 ; 45 : 67-72.

Patel C, Ghanim H, Ravishankar S, et al. Prolonged reactive oxygen species generation and nuclear factor-kappaB activation after a high-fat, high-carbohydrate meal in the obese. J Clin Endocrinol Metab 2007 ; 92 : 4476-9.

Patel S, Puranik R, Nakhla S, et al. Acute hypertriglyceridaemia in humans increases the triglyceride content and decreases the anti-inflammatory capacity of high density lipoproteins. Atherosclerosis 2009 ; 204 : 4248.

Poppitt SD. Postprandial Lipaemia, Haemostasis, Inflammatory Response and other 
Emerging Risk Factors for Cardiovascular Disease: The Influence of Fatty Meals. Curr Nutr Food Sci $2005 ; 1$ : 23-34.

Poppitt SD, Keogh GF, Lithander FE, et al. Postprandial response of adiponectin, interleukin-6, tumor necrosis factor-alpha, and Creactive protein to a high-fat dietary load. Nutrition 2008 ; 24 : 322-9.

Preli RB, Klein KP, Herrington DM. Vascular effects of dietary L-arginine supplementation. Atherosclerosis $2002 ; 162: 1-15$.

Ridker PM, Rifai N, Rose L, Buring JE, Cook NR. Comparison of C-reactive protein and low-density lipoprotein cholesterol levels in the prediction of first cardiovascular events. $N$ Engl J Med 2002 ; 347 : 1557-65.

Rousseau A, Sevin C, Comité de pilotage D.E. S.I.R. Données Epidémiologiques sur le Syndrome d'Insulino-Résistance. 2009 [cited 21/10/2010]; Available from : http ://ifr69. vjf.inserm.fr/ desir/index.html.

Sattar N. Inflammation and endothelial dysfunction: intimate companions in the pathogenesis of vascular disease? Clin Sci (Lond) $2004 ; 106: 443-5$.

Schwartz R, Osborne-Lawrence S, Hahner L, et al. C-reactive protein downregulates endothelial NO synthase and attenuates reendothelialization in vivo in mice. Circ Res 2007 ; 100 : 1452-9.

Sies H, Stahl W, Sevanian A. Nutritional, dietary and postprandial oxidative stress. J Nutr $2005 ; 135$ : 969-72.
Tsai WC, Li YH, Lin CC, Chao TH, Chen JH. Effects of oxidative stress on endothelial function after a high-fat meal. Clin Sci (Lond) $2004 ; 106$ : 315-9.

Tsakiris S, Parthimos T, Parthimos N, Tsakiris $\mathrm{T}$, Schulpis KH. The beneficial effect of Icysteine supplementation on DNA oxidation induced by forced training. Pharmacological Research 2006 ; 53 : 386-90.

Tushuizen ME, Nieuwland R, Scheffer PG, Sturk A, Heine RJ, Diamant M. Two consecutive high-fat meals affect endothelialdependent vasodilation, oxidative stress and cellular microparticles in healthy men. Thromb Haemost 2006 ; 4 : 1003-10.

van Oostrom AJ, Sijmonsma TP, Verseyden C, et al. Postprandial recruitment of neutrophils may contribute to endothelial dysfunction. I Lipid Res 2003; 44 : 576-83.

van Oostrom AJ, Rabelink T], Verseyden C, et al. Activation of leukocytes by postprandial lipemia in healthy volunteers. Atherosclerosis $2004 ; 177: 175-82$.

Vogel RA, Corretti MC, Plotnick GD. Effect of a single high-fat meal on endothelial function in healthy subjects. Am / Cardiol 1997 ; 79 : 350-4.

Vogel RA, Corretti MC, Plotnick GD. The postprandial effect of components of the Mediterranean diet on endothelial function. I Am Coll Cardiol 2000 ; 36 : 1455-60.

Wang L, Gill R, Pedersen TL, Higgins LJ, Newman JW, Rutledge JC. Triglyceride-rich lipoprotein lipolysis releases neutral and oxidized FFAs that induce endothelial cell inflammation. J Lipid Res 2009 ; 50 : 204-13.

Wautier JL, Boulanger E, Wautier MP. Postprandial hyperglycemia alters inflammatory and hemostatic parameters. Diabetes Metab 2006 ; 32 Spec No2: : 2S34-S12.

West SG, Hecker KD, Mustad VA, et al. Acute effects of monounsaturated fatty acids with and without omega- 3 fatty acids on vascular reactivity in individuals with type 2 diabetes. Diabetologia 2005 ; 48 : 113-22.

West SG, Likos-Krick A, Brown P, Mariotti F. Oral L-arginine improves hemodynamic responses to stress and reduces plasma homocysteine in hypercholesterolemic men. J Nutr $2005 ; 135: 212-7$.

Westphal S, Kastner S, Taneva E, Leodolter A, Dierkes I, Luley C. Postprandial lipid and carbohydrate responses after the ingestion of a casein-enriched mixed meal. Am / Clin Nutr $2004 ; 80$ : 284-90.

Westphal S, Taneva E, Kastner S, et al. Endothelial dysfunction induced by postprandial lipemia is neutralized by addition of proteins to the fatty meal. Atherosclerosis 2006 ; 185 : 313-9.

Wu G, Meininger CJ. Arginine nutrition and cardiovascular function. J Nutr $2000 ; 130$ : 2626-9.

Wu G, Fang YZ, Yang S, Lupton JR, Turner ND. Glutathione metabolism and its implications for health. J Nutr 2004 ; 134 : 489-92. 